\title{
Reduction of referral to assessment time for an older adults community mental health team
}

\author{
Chun Chiang Sin Fai Lam \\ South London and Maudsley NHS Foundation Trust
}

\begin{abstract}
Anecdotal evidence suggested that waiting times within the older adults community mental health team (OA CMHT) had been increasing over time. An assessment and evaluation was indicated to ensure best quality care was provided for patients.

A comparison was made between waiting times in January to December 2011 compared with August 2013 to July 2014. In 2011 the mean number of days until initial assessment from the point of referral was 12 days for routine cases, and 3.6 days for urgent cases. The re-audit showed the number of days increased to 15.89 days for routine cases, and 9.81 days for urgent cases.
\end{abstract}

Contributory factors were reviewed, and it was felt that to address this problem, a duty worker role was necessary. The role of the duty worker was divided into triaging and allocating work. The triaging process was to ensure all urgent cases were highlighted early and acted upon.

The duty worker's role was also to gather sufficient information from the referrer, to reduce the risks of inadequate knowledge delaying assessment. In addition, the allocating process required the duty worker to designate a clinician in charge of the case upon receipt of referral. This ensured that clinicians were able to offer the earliest possible appointment slot for the initial assessment, and thus reduce waiting times.

Following implementation, findings from September 2014 to February 2015 showed an improvement in average waiting times, as well as an improvement in the percentage of assessments reviewed within previously set standards. For routine reviews, the mean time until assessment was 10.68 days. For urgent reviews, the mean time until initial assessment was 6.8 days. However, it was noted that majority of urgent reviews were still not being reviewed in time.

The outcomes of this study demonstrated an improvement of both waiting times, and percentage of patients being seen within set standards following a single intervention. In the current climate of cost efficiency savings, it is important for services to continue to find ways to streamline and improve upon current practices, to ensure the best outcome for patients.

\section{Problem}

Anecdotal evidence suggested that waiting times within the $O A$ $\mathrm{CMHT}$ had been increasing over the last year. An assessment and evaluation was indicated to ensure best quality care was provided for patients.

\section{Background}

A Royal College of Psychiatrists briefing, "Mental health and the economic downturn: National priorities and NHS solutions, November 2009",[1] identified that during periods of financial difficulties, there is a risk of waiting lists growing. In this context, it was felt appropriate to review the waiting times for first appointments within a busy London OA CMHT.

The OA CMHT that was assessed provided a service covering the south area of the London Borough of Croydon. It managed a caseload of about 400 patients, aged 65 and over. Patients had mental health disorders which included, but was not limited to, dementias, mood disorders, and delusional disorders.
Referrals into the service most commonly came from GPs, general hospitals, and social services. The team was multidisciplinary in nature, including doctors, community psychiatric nurses, occupational therapists, support workers, social workers, and medical administrators.

All referrals were designated as urgent or routine. The definition of an urgent referral was if there was an imminent risk of serious harm to self, an imminent risk of serious harm to others, or if the referrer stated it was as such.

An outcome was also measured for each referral. A referral was either accepted and seen, accepted but discharged without being seen, or rejected. Although reasons for patients being discharged without being seen or being rejected are important, as it did not fall into the scope of service waiting times, it was not reviewed in this project.

The previous set standard within the OA CMHT was that all routine referrals should be seen within 15 working days of referral, and all urgent referrals within three days. 


\section{Baseline measurement}

An audit of data was conducted previously in the $\mathrm{OA} C M H T$, using data from January to December 2011. In total, 180 patients were referred and seen by services.

The data collected at that point showed that, of all 151 routine cases, $116(76.8 \%)$ were seen within the 15 day work period, and 19 of 29 urgent cases $(65.5 \%)$ were seen within three working days. The mean number of days until initial assessment was 12 days for routine cases, and 3.6 days for urgent cases. The overall average waiting time was 10.65 days, and $75 \%$ (135 of 180 ) of all assessed cases were seen within the set standards.

Data collection was repeated using information gathered from August 2013 to July 2014. In total there were 318 referrals. Two hundred and fifty four $(79.9 \%)$ were accepted and assessed, 48 $(15 \%)$ were rejected, and $16(5 \%)$ were initially accepted but were not seen and later discharged.

During this period, of all 221 assessed routine cases, 144 (65.2\%) were seen within 15 working days, and four of 36 urgent cases $(11.1 \%)$ were seen within three days. In total, $57.6 \%$ (148 of 257 ) of all assessed cases were seen within the previous set standards.

Further analysis of the data showed various reasons for patients not being seen within the requisite time.

1. Delays secondary to the service user contributed to $33.9 \%$ (37 of 109) of all delays in assessment. These were delays due to patients being too physically ill for assessment, requests from patients and/or carers, patients refusing to engage, or inability to communicate with the patient

2. Delays secondary to the NHS provider contributed to $16.5 \%$ (18 of 109) of all delays in assessment. This included inadequate information in the referral causing a delay in assessment, ongoing engagement with other mental health services, and delays due to staffing issues within the team (eg emergencies, next available appointment, sickness, and leave)

3. Unknown causes of delays contributed to $49.5 \%$ (54 of 109) of all delays in assessment. Unknown causes were attributed when no reason for delay was documented within patients' case notes

See appendix for the cause of delays in patients being seen (figure 1).

Further analysis showed that $8.3 \%$ of cases were not seen due to inadequate information. It was expected that all referrals should have a past medical history and current medication list. The data from August 2013 to July 2014 showed that $58 \%$ (156 of 269) of all referrals did not have this minimum prerequisite.

Part of the baseline measurement was to assess the average waiting time to be seen by a clinician with the $\mathrm{OA} C M H T$ from receipt of referral. In keeping with an intention to treat analysis, all patients who were seen were included in the analysis. Patients who were not seen by the service were not included in the analysis, as it was not possible to capture their waiting time following the above definition.

Of the routine reviews, the mean number of days until being seen was 15.89 days. Of the urgent reviews, the mean number of days until being seen was 9.81 days. The total average time was 15.04 days.

\section{Design}

Baseline measurements suggested deterioration from previous standards. Discussions were held to assess possible contributory factors leading to the increase in waiting times compared to 2011. Contributory members included team managers, consultants, community psychiatric nurses, and nurse specialists.

In 2011 a duty system for referrals was in place, where a member of staff triaged all cases being referred on a daily basis. Due to staff shortage the system was not sustainable, however, and it was changed by 2013 to a weekly meeting where new referrals were discussed. On occasions, team meetings were cancelled due to holidays, and this caused referrals to be reviewed up to five working days later. The lack of information in some referrals had also led to a direct increase in waiting time delay, as team members gathered the information. However, if there were cases marked urgent by the referrer, the administration staff would bring these to senior clinicians to discuss review.

\section{Strategy}

Since the initial data collection was conducted, changes had occurred within the service. The two OA CMHTs within the borough (north and south) merged to form a single working unit. Although it led to a net loss of staff (equivalent of eight whole time equivalent (WTE) staff), this allowed a consolidation of manpower resources which permitted the daily duty system to be reimplemented. A standard operating procedure was produced by the clinical nurse specialist in the team, and a pilot trial was implemented. The reintroduction of the duty worker served to address many of the factors leading to delays in assessment.

The role of the duty worker was divided into triaging and allocating work. The triaging process was to ensure that all referrals were reviewed, and if there were cases of concern, the duty worker could discuss this with the senior clinician on site. This ensured that urgent cases were highlighted early and acted upon. The duty worker's role was also to gather sufficient information from the referrer, to reduce the risk of inadequate knowledge delaying assessment. If a case was referred as urgent, the duty worker would attempt to obtain collateral information on the same day, and if required, to attend immediately.

The allocating process required the duty worker to designate a clinician in charge of the case upon receipt of referral. This ensured that clinicians were able to offer the earliest possible appointment slot for the initial assessment, and thus further reduce waiting times. 


\section{Results}

Initial findings from the September 2014 to February 2015 period of implementation suggested an improvement in waiting times. In total 297 referrals were received, of which 218 were accepted and seen.

During this period, $85.1 \%$ (143 of 168 ) of all assessed routine cases were seen within 15 working days, and $36 \%$ (18 of 50 ) of urgent cases were seen within three days. In total, $73.9 \%$ (161 of 218) of all assessed cases were seen within the previous set standards.

Of the routine reviews, the mean number of days until being seen was 10.68 days. Of the urgent reviews, the mean number of days to being seen was 6.8 days. In total, average waiting times had reduced to 12.7 days.

Review of the results suggested that there was an improvement in average waiting times and percentage of assessments reviewed. However, the majority of urgent reviews were still not being reviewed in time.

See appendix for percentage of cases reviewed within set standards (figure 2), and average waiting time from receipt of referral to assessment (figure 3 )

The 32 urgent cases which breached the previous set standards were reviewed. Seven cases were delays secondary to service users, and 25 cases were due to unknown causes. These cases were individually assessed, and it was found that in 18 of these cases, although the referrer had felt the case was urgent, the triaging duty worker had downgraded the status. In each occasion, the duty worker had contacted the referrer or main carer following receipt of referral, and had felt that the risk which presented did not warrant an urgent review. Seven cases were deemed urgent but were not seen in the allocated time.

This suggested that the threshold for what was deemed urgent by referrers and the OA CMHT differed, and suggests scope for further improvement in the future for matching expectations between services.

See supplementary file: ds6481.pdf - "Appendix (Figures)"

\section{Lessons and limitations}

This report demonstrated the ability to improve waiting times by merging teams, and implementing rapid triaging and allocation of referrals. In spite of the loss of staff during this period, the OA $\mathrm{CMHT}$ had been able to produce better outcomes. This was likely due to consolidation of human resources which reduced the duplication of similar services. This assumption does not take into account other human factors, which may include the dedication of the remaining staff and the impact upon the quantity of cases they need to manage, and quality of care provided. This would be a possible aspect for further assessment in the future, along with looking into matching referral expectations of the service.
Limitations to this study included looking at historical data for the purpose of comparisons. The data being obtained is reliant on the quality of the notes being input into the electronic system. There is a possibility of notes being delayed or not being adequately completed, which would have an effect on the data collected. Furthermore, the initial audit report in 2011 provided only the final findings, and there was less information in the methodology, which may make it less valid as a comparative point.

Moreover, there was assumed causality that the reduction in working time was linked to the intervention, and there could potentially have been other social or institutional factors that influenced the outcome. During this period of study, the OA CMHT underwent a significant period of recruitment, turnover, and attrition. Consideration was given towards using working time equivalent of staffing levels and correlation to waiting times for further clarity, but due to the constant flux in staff members, a consistent measurement was difficult to formulate.

\section{Conclusion}

Waiting times within the NHS are often used in society as a benchmark of good quality care. In 2014, the Chief Medical Officer, Dame Sally Davies, recognised the need for waiting time targets and improved access to mental health services for patients. A delay in obtaining treatment for various mental health conditions is linked with a poorer prognosis, and causes increased distress to both the patient and their family.

The outcomes of this study have demonstrated an improvement of both waiting times and percentage of patients being seen within set standards following a single intervention. In the current climate of cost efficiency savings, it is important for services to continue to find ways to streamline and improve upon current practices, to ensure the best outcome for patients within the limitations of financial and manpower restrictions.

\section{References}

1. Mental health and the economic downturn: National priorities and NHS solutions. Royal College of Psychiatrists November 2009. http://www.rcpsych.ac.uk/files/pdfversion/OP70.pdf

\section{Declaration of interests}

Nothing to declare

\section{Acknowledgements}

Raziah Nanuck, Dr Raisha Nurani, Dr Peter Medland, Dr Maria Moisan, Dr Anita Duggal, Dr Dipankar Bandy

\section{Ethical approval}

Ethical approval not applied for project. 
BMJ Quality Improvement Reports

Clinical governance approval for this project was given by the South London and Maudsley NHS Foundation Trust. 